\title{
Two Stage Total Hip Revision in Treatment of Chronic Infection of Hip Prosthesis
}

\author{
Sayed El-Etewy Soudy* and Mohsen Fawzy Omar
}

Orthopedic Surgery Department, Faculty of Medicine, Zagazig University, Egypt

*Corresponding author: Soudy SEE, Assistant Professor, Orthopedic Surgery Department, Faculty of Medicine, Zagazig University, Egypt, Fax: 1005624590, Tel: 20-553668921; E-mail: yousufmmkh@gmail.com

Received date: Oct 26, 2016; Accepted date: Nov 04, 2016; Published date: Nov 08, 2016

Citation: Soudy SEE, Omar MF (2016) Two Stage Total Hip Revision in Treatment of Chronic Infection of Hip Prosthesis. J Clin Exp Orthop 2: 25. doi: $10.4172 / 2471-8416.100025$

Copyright: (C) 2016 Soudy SEE, et al. This is an open-access article distributed under the terms of the Creative Commons Attribution License, which permits unrestricted use, distribution, and reproduction in any medium, provided the original author and source are credited.

\section{Introduction}

The deep chronic infection of arthroplasty implants is a serious problem and requires great effort and cost for its treatment. Pharmacological and surgical options of treatment have been reported in the literature. The issues related to this situation are the route of administration and duration of antibiotics, stages of surgery, and the time of re-implantation of the new prosthesis [1,2]. There are different protocols of antibiotics regimens which range from no antibiotics $[3,4]$ to a long course of parenteral antibiotic for 9 weeks, followed by oral administration. The costing, and side effects or toxicity of the antibiotic are the causes of these controversies. The surgical solutions also vary from resection arthroplasty (Girdlestone) [2-5], retention of the prosthesis with debridement [6], onestage revision [7], and two-stage revision with hand-made cement spacer loaded with antibiotic [8], or with prefabricated spacer prosthesis [9].

It is well accepted that removing the foreign bodies is mandatory to eradicate chronic infections around the implant [6]. The resection arthroplasty technique is associated with loss of function and limited to non-cooperative patients and who are unfit to have an additional reconstructive surgery. The advantage of one-stage revision is the low cost, decrease period of patient recumbence, and preserves patient function. Preoperative identification of the infecting organism is mandatory and the antibiotics loaded to bone cement can be chosen according to culture sensitivity [1-3]. The mid- and longterm results of this cemented revision arthroplasty reported poor cement interdigitation [4,5]. Added to this the difficult reconstruction in patients with severe bone loss, and lower eradication rate in one-stage revision arthroplasty when compared to two-stage revision [6]. Due to all previous reports, the two-stage revision has become the standard approach for higher eradication rate of resistant organisms $[7,8]$.

The two stages of revision are: first, removal of the infected prosthesis and bone cement, generous debridement to remove all infected and necrotic tissues, and implantation of a cement spacer. Open biopsy for culture sensitivity and histopathology are taken and antibiotics according to its result. During the second stage after 8-12 weeks consists of removal of the spacer, and implantation of the new prosthesis covered by a systemic antibiotic therapy. Many authors reported good results and fewer complications after the second operation when using the cement spacers [9-13]. Several factors can affect the results of two-stage technique as the general condition of the patients, the number and type of organisms, antibiotics sensitivity, and extension of infection.

The articulated spacer PROSTALAC (prosthesis of antibioticloaded acrylic cement) have shown good results in the latest reports of literature [11-14]. This system was available only in North America, but recently, different sizes of preformed spacers (Spacer-G1) have become available worldwide with good results [15-17].

The aim of this prospective study is to evaluate the results of two-stage revision arthroplasty technique using antibioticloaded spacers for the treatment of deep periprosthetic infections of hip prosthesis.

\section{Patients and Methods}

Thirty patients 20 males and 10 females with a mean age of 61 years (range: $38-72$ ) had infected total hip arthroplasty (THA) were treated using two-stage revision operations. All the operations were performed in our institution in the period from May 2009 and May 2014. The diagnosed of infection was made through clinical, laboratory, and radiological evaluation. Clinically, the general health status, patient history, pain and wound condition were recorded. The laboratory investigations were ESR (erythrocyte sedimentation rate), CRP (C-reactive protein), WBC (white blood cell), and hip aspiration under complete aseptic conditions, or from fistula. Pre-operative plain $\mathrm{x}$-rays included anterior-posterior view of the pelvis and lateral view of the hip joint were done to evaluate loosening, migration, or presence of any hidden sinus. The $99 \mathrm{mTc}$-leukocyte-labeled scintigraphies were done for 20 patients. The remaining ten patients had sinuses discharging pus.

The cause of revision was septic total hip prosthesis. The $\mathrm{ZMR}^{\circledR}$ modular cement less distally locked prosthesis was used in all cases. The mean duration of the follow-up was 36 months (range: 24-60), and six cases were lost in follow-up and excluded from the study. All the operations were performed in our institution and by the same surgical team. The infected 
prosthesis was total hip in 18 cases and hemiarthroplasty in twelve cases (10 bipolar, and 2 Thompson) (Figure 1).

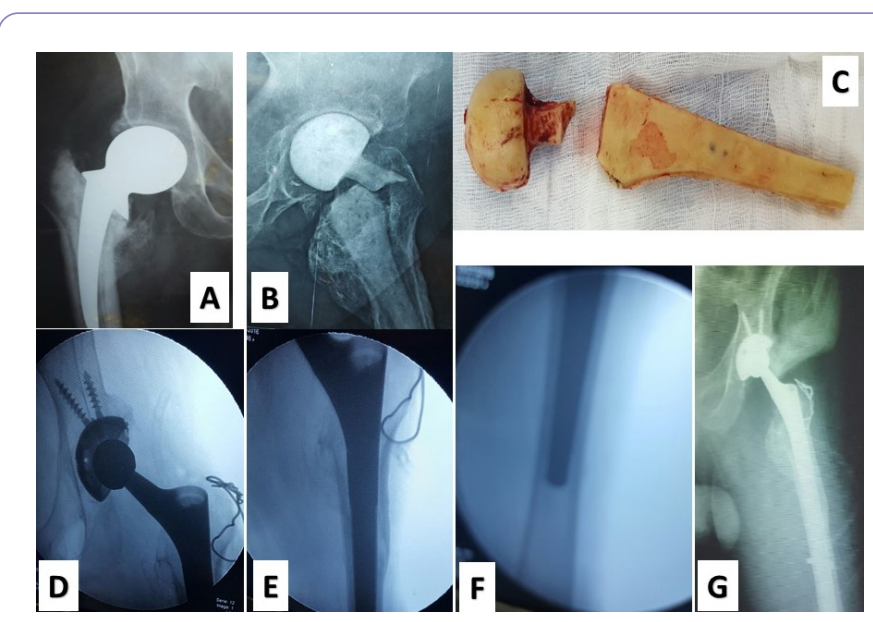

Figure 1: Male patient 62 years old with infected Thompson prosthesis; A) preoperative X-ray; B) X-ray before the second stage with Brocken cement spacer; $C$ ) intraoperative photo after extraction of spacer; $D, E$ and $F$ ) intraoperative radiology after stem application to insure position and bone integrity; $\mathrm{G})$ postoperative $\mathrm{X}$-ray after revision cementless thr with calcar replacement long stem.

As the prognosis can be affected by the patient's risk factors, the Cierny-Mader [18] physiological classification was used for

Table 1: Cierny-Mader Staging System. preoperative evaluation (Table 1). Operative technique: All operations were done in lateral position using lateral approach. Extended trochanteric osteotomies were needed in 22 cases of 30 , but the remaining 8 prostheses were loose. Complete excision of the sinus was performed in 10 cases. Complete removal of the prosthesis including plug and cement followed by thorough debridement of all potentially infected soft tissue. In all cases, intraoperative open samples were taken for culture, from three sites separately (joint capsule, acetabulum, and femoral canal). Copious irrigation using a pulsed lavage system. Using acetabular and femoral reamers, both the acetabulum and femoral canal were reamed to get rid of all infected and necrotic tissues and to contain the antibiotic loaded cement spacer.

After completing the procedure, the wound was closed over suction drain and broad spectrum antibiotics of third generation cephalosporin were administrated until the result of culture sensitivity to give according to the organism sensitivity. The drain was removed on the second day after the operation, and then the patients were allowed to walk with toe-touch weight bearing through the duration between the two stages. Through the interval period, the antibiotic therapy duration ranged from 4 to 6 weeks followed by one week of antibiotic discontinuation at least. General and local conditions and laboratory tests were checked regularly. The lab investigations that were performed to monitor the response to infection are the ESR and CRP.

\section{Anatomic type}

Stage 1: Medullary osteomyelitis

Stage 2: Superficial osteomyelitis

Stage 3: Localized osteomyelitis

Stage 4: Diffuse osteomyelitis

Physiologic class

A host: Normal host

B host: Systemic compromise (Bs)*

Local compromise (BI)*

Systemic and local compromise (Bls) ${ }^{*}$

C host: Treatment worse than the disease

Systemic or local factors that affect immune surveillance, metabolism, and local vascularity

\begin{tabular}{|c|c|}
\hline Systemic (Bs) & Local (BI) \\
\hline Malnutrition & Chronic lymphedema \\
\hline Renal or hepatic failure & Venous stasis \\
\hline Diabetes mellitus & Major-vessel compromise \\
\hline Chronic hypoxia & Arteritis \\
\hline Immune disease & Extensive scarring \\
\hline Malignancy & Radiation fibrosis \\
\hline Extremes of age & Small-vessel disease \\
\hline Immunosuppression or neuropathy & Complete loss of sensation \\
\hline Immune deficiency & Tobacco abuse \\
\hline
\end{tabular}


In this work we used cement handmade spacer in all cases. The shape of the space was like the unipolar hemiarthroplasty (Figure 2).

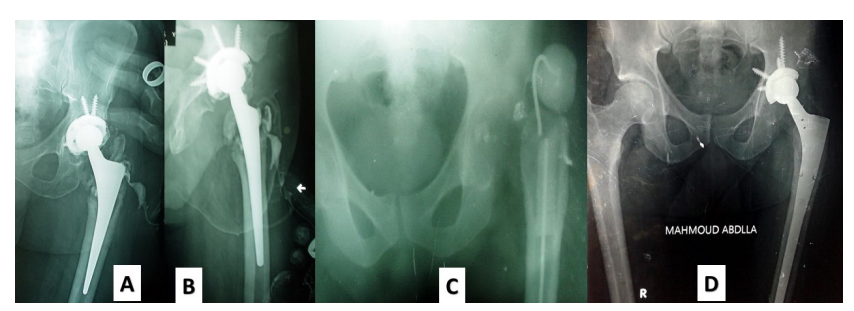

Figure 2: Male patient 38 years old with infected total hip; $A$ and B) X-ray with sinogram showing infected total hip; C) Xray before second operation; D) X-ray 3 years after revision with cement less long stem.

The second stage revision was performed once the CRP or ESR readings are normal. Hip joint aspiration needed in 10 cases of 30 before second stage. If the patients shifted from B-host to Chost during the interim period, a resection arthroplasty was performed according to Cierny-Mader [18].

In the second stage operation another debridement and irrigation using the pulsed lavage system after removal of the spacer. A parenteral prophylactic antibiotics were administrated until the results of intra-operative samples were proven to be negative. In two cases, the results of the intra-operative samples were positive and the recommended antibiotic therapy was administered for 3 weeks. Partial weight bearing was allowed in the first using axillary crutches 6 weeks postoperative and full weight bearing from 8 to 9 weeks with contralateral elbow crutch. The patients regularly underwent clinical, radiographic and laboratory tests. The functional outcome was evaluated before the first stage operation and at the time of the latest follow-up using the Harris Hip Score [19]. Other follow-up parameters are serial plain radiographs, ESR, CRP and full blood counts (FBC).

Radiographic evaluation was done using Engh et al. [20] system. Gruen et al. [21] for radiolucencies in the boneprosthesis interface, and DeLee and Charnley [22] for acetabular evaluation was performed. Acetabular inclination angle of $35^{\circ}$ to $55^{\circ}$ was considered accepted. Implant migration, complete radiolucent line at the implant bone interface, or screws breakage were defined as signs of cup loosening. Brooker et al. [23] system was used for heterotopic ossification grading.

\section{Results}

After the first operation, the follow-up laboratory parameters to exclude recurrence were done ESR, CRP, WBC count. Eighteen patients were clinically improved and ESR, CRP normal value. Five patients needed aspiration from the hip and revealed no growth of organisms. During the interim period, five stems dislocated and seven breakage. Five patients with stem breakage needed second stage after 7 weeks and reimplantation of spacer. In two patients the spacer removed and left girdle stone until second stage operation. The inflammatory parameters (ESR
$+\mathrm{CRP}+\mathrm{WBC}$ ) reached normal values in 18 patients after 4 weeks of removal of prosthesis. Other twelve patients delayed up to 8 weeks until normalization of inflammation parameters.

The reimplantation of the new cement less revision implant of revision was performed after a mean of 12 weeks (range: 8-24) after normalization of the inflammatory parameters. The samples that have been taken at the time of spacer removal and reimplantation were negative for bacterial growth in 24 patients. In six patients the samples were positive but no evidence of recurrence of infection was noted in these cases during follow-up. In the patients with positive sample results antibiotic treatment was administrated according to sensitivity for four to six weeks. After the second operation, clinical evaluation and laboratory tests were done for all patients regularly and through the duration of follow-up there was no recurrence of infection detected.

After the second stage operation, none of the patients had a dislocation of the prosthesis. Two patients had stem subsidence of 5-mm after 6 months from surgery, but the stems were stable and no clinical problems without additional subsidence during the follow-up period. The rest of stems had good fixation and bone-ingrowth.

Clinically, the Harris hip score was improved from a preoperative mean of $43(28-62)$ to a postoperative mean of 87 (79-96) at final follow-up.

Postoperative follow-up X-rays (AP, Lateral views of hip joint with AP view of pelvis) were evaluated monthly for 6 months, and 6 monthly tell the last follow-up. There was no postoperative dislocations. Brooker grade II heterotopic ossification occurred in five patients (16.7\%), and grade III in two patients $(6.5 \%)$. The acetabular inclination was within the accepted range 40-50 o in all hips. The femoral stem was in neutral position in 24 stems (79\%), 4 stems (14.5\%) in varus, and valgus position in 2 stems (6.5\%).

According to Engh's criteria, 24 of 30 stems were stable and had bony ingrowth with no significant subsidence, 6 were stable fibrous. In six hips, there was $1 \mathrm{~mm}$ radiolucent line of acetabular component in 2 zones (21\%), but these lucencies were not progressive. Gruen zones for femoral stem had revealed four stems with $3 \mathrm{~mm}$ radiolucency 3 zones but stems were stable.

\section{Discussion}

The results of our treatment protocol are satisfactory. All deep periprosthetic infections were eradicated, and forty of 41 patients had final reimplantation. For the deep periprosthetic infection, the two-stage revision hip arthroplasty is considered the gold standard for treatment [24]. The high cost of the two operations in contrast to one-stage revision arthroplasty is considered a disadvantage [25].

Hanssen and Rand [26] using antibiotic-loaded cement reported $83 \%$ success rate, and $60 \%$ success rate when using non antibiotic-loaded cement. Another two studies carried out by Raut et al. $[25,27]$ on 57 hips with draining sinuses revised in one stage operation. They reported $86 \%$ success rate at 7.4 
years average follow-up period, and $93.4 \%$ success rate in patients with gram-negative infections after 8 years average follow-up.

Many authors reported lower rate of eradication in one-stage revision operations than in two-stage operations for revision hip arthroplasty with highly virulent organisms that cause the infection. The cement less two-stage revisions of chronic infection of total hip lacks the advantage of local antibiotic loaded bone cement $[28,29]$. Authors who recommend one stage revision propose lower virulence organism and antibioticloaded cement should be used and the patients do not have severe bone loss.

Before final reimplantation of the revision prosthesis it is a challenge for the surgeon to get prove that infection has been completely eradicated and that may be difficult to diagnose. In this work, the two-stage revision arthroplasty was done for all patients doing the same protocol for follow-up in interval between the two operations. The protocol included regular (ESR, CRP, AND WBC count), hip aspiration in doubt, the 99mTc bone scan, and Cierny-Mader classification for the general condition and physiological stage. The patient's risk factors is very important to be taken into consideration. Cierny et al. [18] using an osteomyelitis classification system designed to stratify treatment selection according to patient risk factors reported the results of treatment of 43 patients with periprosthetic infection. In their study the survival rate of the implant in patients with three or more morbidities was recorded $0 \%$ after follow-up of two years. They concluded that the two most important variables in predicting outcomes in patients with prosthetic infections are the duration of infection and the host condition [30].

In this study, there were 12 patients class B-host having three or more morbidity, the final reimplantation of the prosthesis was performed with no recurrence of the infection during follow-up period [31,32]. As the resistant organisms has been increasing over the recent decades, the surgery complications increased in comparison to non-resistant organisms causing the infection. Volin et al. [33] used two-stage revision arthroplasty in the treatment of two groups of patients (a methicillin-sensitive group and a methicillin-resistant group) and compared the outcomes that was similar in the two groups.

In this study, we have 10 of 30 patients (33\%) had a methicillin-resistant Staphylococcus, and no difference was found between the one-spacer group and the two-spacer group. Reinfection can occur in a period up to 5 years after operation, but usually observed within the first year after operation [34-36]. In this work the cement handmade spacer in all cases to give the shape of unipolar hemiarthroplasty. We used a high dose of vancomycin in the cement. Springer et al. [37] evaluated the potential complications resulting from the use of high doses of antibiotic loaded to bone cement spacer in two-stage resection arthroplasty of the knee. They found no systemic sideeffects in the patients clinically. In this work we had no evidence of disease caused by antibiotics systemic side-effects.

The sensitivity and specificity of $99 \mathrm{mTc}$ bone scan was evaluated by Pelosi et al. [38] and reported 95.6\%. Also Larikka et al. [39] reported improvement specificity of leucocyte imaging in the diagnosis of infected hip prostheses. In this study $99 \mathrm{mTc}$ bone scan was utilized in cases of confusion in the diagnosis. From the limitations of this study, the small number of cases and lake of control group managed with one-stage revision.

\section{Conclusion}

After evaluation of 30 cases we consider the use of two-stage revision protocol for treatment of infected hip arthroplasty is successful in eradication of the infection and stable joint reconstruction. In the strategy of infection eradication, the success rate depends on good protocol considering the risk factors of the patients and the proper evaluation of the diagnostic tests. The two-stage revision has the advantage of the assessment of the response to the selected antibiotics and the chance to change the antibiotic in the second stage if the organism changed or resistant. The possibility using cement less stems increased after eradication of infection.

\section{References}

1. Goulet JA, Pellicci PM, Brause BD, Salvati EM (1988) Prolonged suppression of infection in total hip arthroplasty. J Arthroplasty 3: 109-116.

2. Canner GC, Steinberg ME, Heppenstall RB, Balderston R (1984) The infected hip after total hip arthroplasty. J Bone Joint Surg Am 66: 1393-1399.

3. Bourne RB, Hunter GA, Rorabeck CH, Macnab JJ (1984) A six-year follow-up of infected total hip replacements managed by Girdlestone's arthroplasty. J Bone Joint Surg Br 66: 340-343.

4. Dohmae Y, Bechtold JE, Sherman RE, Puno RM, Gustilo RB (1988) Reduction in cement-bone interface shear strength between primary and revision arthroplasty. Clin Orthop Relat Res 236: 214-220.

5. Lie SA, Havelin LI, Furnes ON, Engesaeter LB, Vollset SE (2004) Failure rates of 4762 revision total hip artrhroplasties in the Norwegian Arthroplasty Register. J Bone Joint Surg Br 86: 504-509.

6. Garvin KL, Hanssen AD (1995) Current concepts review. Infection after total hip arthroplasty. Past, present, future. J Bone Joint Surg Am 7: 1576-1588.

7. EARRS Annual Report 2006. EARSS. The European Antimicrobial Resistance Surveillance System. 2009 February 17.

8. Evans R, Clyburn T, Joseph J (2008) Orthopedic infection: community-acquired and hospital-acquired methicillin-resistant Staphylococcus aureus (MRSA) AAOS patient safety committee scientific exhibit.

9. Younger ASE, Duncan CP, Masri BA, McGraw RW (1997) The outcome of two-stage arthroplasty using a custom-made interval spacer to treat the infected hip. J Arthroplasty 12: 615-623.

10. Younger ASE, Duncan CP, Masri BA (1998) Treatment of infection with segmental bone loss in the proximal part of the femur in two stages with use of antibiotic-loaded interval prosthesis. J Bone Joint Surg Am 80: 6069.

11. Masri BA, Duncan CP, Beauchamp CP (1998) Long-term elution of antibiotics from bone-cement: $A n$ in vivo study using the prosthesis of antibiotic-loaded acrylic cement (PROSTALAC) system. J Arthroplasty 13: 331-338. 
12. Haddad FS, Masri BA, Garbuz DS, Duncan CP (1999) The treatment of the infected hip replacement. The complex case. Clin Orthop Relat Res 369: 144-156.

13. Anagnostakos K, Fürst $O$, Kelm J (2006) Antibiotic-impregnated PMMA hip spacers: Current status. Acta Orthop 77: 628-637.

14. Masri BA, Panagiotopoulos KP, Greidanus NV, Garbuz DS, Duncan CP (2007) Cementless two-stage exchange arthroplasty for infection after total hip arthroplasty. J Arthroplasty 22: 72-78.

15. Magnan B, Regis D, Biscaglia R, Bartolozzi P (2001) Preformed acrylic bone cement spacer loaded with antibiotics: use of twostage procedure in 10 patients because of infected hips after total replacement. Acta Orthop Scand 72: 591-594.

16. D'Angelo F, Negri L, Zatti G, Grassi FA (2005) Two-stage revision surgery to treat an infected hip implant. A comparison between a custom-made spacer and a pre-formed one. Chir Organi Mov 90: 271-279.

17. Meani E, Romanò CL, Crosby L, Hofmann G (2007) Infection and local treatment in orthopaedic surgery. Berlin Heidelberg (GER): Springer-Verlag.

18. Cierny G, Mader JT, Pennick H (1985) A clinical staging system of adult osteomyelitis. Contemp Orthop 10: 17-37

19. Harris WH (1969) Traumatic arthritis of the hip after dislocation and acetabular fractures: treatment by mold arthroplasty. J Bone Joint Surg Am 51: 737-755.

20. Engh CA, Bobyn JD, Glassman AH (1987) Porous coated hip replacement. The factors governing bone ingrowth, stress shielding, and clinical results. J Bone Joint Surg 69: 45-55.

21. Gruen TA, McNeice GM, Amstutz HC (1979) Modes of failure" of cemented stem-type femoral components: a radiographic analysis of loosening. Clin Orthop 141:17.

22. DeLee JG, Charnley J (1976) Radiological demarcation of cemented sockets in total hip replacement. Clin. Orthop 121: 20-32.

23. Brooker AF, Bowerman JW, Robinson RA (1973) Ectopic ossification following total hip replacement. Incidence and a method of classification. J Bone Joint Surg 55: 1629-1632.

24. Hsieh PH, Shih CH, Chang YH, Lee MS, Shih HN, et al. (2004) Twostage revision hip arthroplasty for infection: comparison between the interim use of antibiotic-loaded cement beads and a spacer prosthesis. J Bone Joint Surg Am 86: 1989-1997.

25. Raut VV, Siney PD, Wroblewski BM (1994) One-stage revision of infected total hip replacements with discharging sinuses. J Bone Joint Surg Br 76: 721-724.
26. Hanssen AD, Rand JA (1998) Evaluation and treatment of infection at the site of a total hip or knee arthroplasty. J Bone Joint Surg Am 80: 910-922.

27. Raut VV, Orth MS, Orth MC (1996) One stage revision arthroplasty of the hip for deep gram negative infection. Int Orthop 20: 12-14.

28. Salvati EA, Chekofsky KM, Brause BD, Wilson PD (1982) Reimplantation in infection: a 12-year experience. Clin Orthop Relat Res 170: 62-75.

29. Fitzgerald RH (1995) Infected total hip arthroplasty: diagnosis and treatment. J Am Acad Orthop Surgeons 3: 249-262.

30. Buchholz HW, Elson RA, Engelbrecht E (1981) Management of deep infection of total hip replacement. J Bone Joint Surg $\mathrm{Br}$ 63: 342-353.

31. Ure KJ, Amstutz HC, Schmalzried TP (1988) Direct-exchange arthroplasty for the treatment of infection after total hip replacement. J Bone Joint Surg Am 80: 961-968.

32. Callaghan JJ, Katz RP, Johnston RC (1999) One-stage revision surgery of the infected hip. Clin Orthop Relat Res 369: 139-143.

33. Volin SJ, Hinrichs SH, Garvin KL (2004) Two-stage reimplantation of the total joint infections: a comparison of resistant and nonresistant organisms. Clin Orthop Relat Res 427: 97-100.

34. Haddad FS, Muirhead-Allwood SK, Manktelow ARJ (2000) Twostage uncemented revision hip arthroplasty for infection. J Bone Joint Surg Br 82: 689-694.

35. Lai KA, Shen WJ, Yang CY, Lin RM, Lin CJ, et al. (1996) Two-stage cementless revision THR after infection. 5 recurrences in 40 cases followed 2.5-7 years. Acta Orthop Scand 67: 325-328.

36. Wang JW, Chen CE (1997) Reimplantation of infected hip arthroplasties using bone allografts. Clin Orthop Relat Res 335: 202-210.

37. Springer BD, Lee GC, Osmon D (2004) Systemic safety of high-dose antibiotic-loaded cement spacers after resection of an infected total knee arthroplasty. Clin Orthop Relat Res 427: 47-51.

38. Pelosi E, Baiocco C, Pennone M, Migliaretti G, Varetto T, et al. (2004) 99mTc-HMPAO-leukocyte scintigraphy in patients with symptomatic total hip or knee arthroplasty: Improved diagnostic accuracy by means of semiquantitative evaluation. J Nucl Med 45: 438-444.

39. Larikka MJ, Ahonen AK, Junila JA, Niemelä O, Hämäläinen $M M$, et al. (2001) Extended combined 99mTc-white blood cell and bone imaging improves the diagnostic accuracy in the detection of hip replacement infections. Eur J Nucl Med 28: 288-293. 